\title{
La división sexual del trabajo. Reflexiones desde el Feminismo Materialista Francés
}

\author{
The sexual division of labor. Insights from French Materialist Feminism
}

\author{
Victoria Estermann \\ victoria.estermann@gmail.com \\ Instituto de Investigaciones en Humanidades y \\ Ciencias Sociales. Facultad de Humanidades y Ciencias \\ de la Educación. Universidad Nacional de La Plata, \\ Argentina
}

Recepción: 31 Julio 2020

Aprobación: 06 Noviembre 2020

Publicación: 01 Septiembre 2021

Cita sugerida: Estermann, V. (2021). La división sexual del trabajo. Reflexiones desde el Feminismo Materialista Francés. Descentrada, 5(2), e152. https://doi.org/10.24215/25457284e152

\begin{abstract}
Resumen: Este artículo se propone reponer las teorizaciones en torno a la división sexual del trabajo desde el feminismo materialista francés, en particular, la propuesta de Danièle Kergoat acerca de las relaciones sociales estructurales. El objetivo es evaluar sus aportes a algunos debates actuales en el interior de los estudios del trabajo, entre los cuales la conciliación entre trabajo doméstico y trabajo asalariado es la más relevante. Una de las principales conclusiones es que dicha perspectiva puede ser útil para analizar las características del mundo del trabajo desde una perspectiva imbricacional, donde los distintos ejes de opresión sean tenidos en cuenta en sus configuraciones específicas.
\end{abstract}

Palabras clave: División sexual del trabajo, Feminismo materialista francés, Danièle Kergoat.

\begin{abstract}
The aim of this article is to rethink the sexual division of labor theorizations from the French materialist feminism perspective, in particular around Danièle Kergoat's proposal about structural social relations. We pretend to evaluate their contributions to some of the current debates within labor studies, among which the reconciliation between domestic and paid work is the most relevant. One of the main conclusions is that this perspective can be useful to analyze the characteristics of the world of labor from an interwined perspective where the different axes of oppression are taken into account in their specific configurations.
\end{abstract}

Keywords: Sexual division of labor, French materialist feminism, Danièle Kergoat.

\section{INTRODUCCIÓN}

Las discusiones sobre trabajo y género en el interior de las ciencias sociales han tenido que enfrentar el desafío de explicar las grandes transformaciones ocurridas en el interior del mercado de trabajo, donde las nuevas formas de organización del capital han originado una situación de fragmentación y heterogeneidad de sectores cada vez más grandes de la clase trabajadora. A esto se le ha sumado la conformación de grandes cadenas globales de cuidado, donde las opresiones de sexo-género, raza y clase se muestran en toda su crudeza. ${ }^{1}$ 
Estos desarrollos dan cuenta de la importancia de tener una perspectiva crítica en ciencias sociales para reflexionar sobre la vinculación entre trabajo doméstico y trabajo asalariado, a fines de poder comprender las configuraciones del mundo del trabajo en un nivel global y de habilitar los debates acerca de las formas en las que las relaciones de producción se configuran e impactan en las vidas de individuos marcados por la clase, la raza y el sexo.

En el marco de estas discusiones, nos interesa abordar la división sexual del trabajo y las relaciones sociales estructurales de género desde la perspectiva del feminismo materialista francés. Creemos que pensar al trabajo como generador de divisiones sexuales y de relaciones de poder en distintas esferas nos permite avanzar en la comprensión de la interrelación entre trabajo doméstico y trabajo asalariado, así como las configuraciones que el segundo presenta en la clase social de las mujeres. ${ }^{2}$

El objetivo de este texto es reponer las teorizaciones en torno a estos dos conceptos, así como las discusiones que se suscitaron de la mano de la socióloga Danièle Kergoat, a fin de evaluar los aportes de estas categorías a algunos debates actuales de la sociología y los estudios del trabajo. Coincidimos con Pasero Brozovich cuando destaca la importancia de esta perspectiva a la hora de "profundizar el análisis de las relaciones sociales desde una mirada crítica feminista para entender en toda su complejidad el lugar del trabajo realizado por las mujeres" (Pasero Brozovich, 2016, p. 2).

En primer lugar, nos detendremos brevemente en la propuesta del feminismo materialista francés para definirla. En un segundo lugar, analizaremos los conceptos de división sexual del trabajo y relaciones sociales estructurales de dominación. Por último, intentaremos reflexionar acerca de los límites y las potencialidades de estos conceptos para pensar la conciliación entre trabajo doméstico y trabajo asalariado.

\section{El Feminismo Materialista Francés y sus discusiones sobre la Clase de las MUJERES}

El feminismo materialista francés (FMF) es una corriente que se desarrolla en Francia, en la década de 1970, al calor del Movimiento social de Liberación de las Mujeres y en estrecha ligazón con él. Tiene su punto de partida en la obra de Simone de Beauvoir y confronta con las lecturas tradicionales del materialismo histórico, retomando para su análisis algunos textos de Engels, en particular El Origen de la familia, de la propiedad privada y del Estado, aunque discutiendo críticamente su postura sobre la división sexual del trabajo (Femenías, 2015).

Nucleado en la revista Questions Féministes, logra elaborar un conjunto teórico con una fuerte homogeneidad y coherencia interna (Curiel y Falquet, 2005; Bolla, 2020) que permite dar cuenta de la opresión de las mujeres en cuanto clase social. Su propuesta teórica se desarrolla alrededor de la idea central de las dimensiones materiales y estructurales de la situación social de las mujeres y los varones, como clases de sexo dialécticamente producidas la una por la otra (Falquet, 2016). El FMF se distingue así de otras propuestas por el carácter radicalmente anti-biologicista del mismo; es decir, estas autoras plantean la clase social de las mujeres como una clase social, en pleno sentido del término, distanciándose radicalmente de las propuestas anglosajonas del sistema sexo-género, que se apoyan sobre la idea de que hay al menos un sustrato natural (el sexo biológico, los órganos sexuales y reproductivos) y que sobre tal base se imprime o codifica posteriormente el género, pensado como cultural.

Con el objetivo de distinguirse del marxismo, las autoras ligadas al FMF plantean que se deben diferenciar los conceptos generales propuestos por el materialismo histórico (clase, explotación, modo de producción, trabajo) y su utilización concreta en los análisis marxistas (por ejemplo, la explicación del modo de producción capitalista). Desplazar estos conceptos les permite poder analizar otros modos de producción y relaciones que coexisten con el capitalismo: la "apropiación” y el sexage formulados por Guillaumin o el "modo de producción doméstico" de Delphy. ${ }^{3}$ 
La propuesta de Kergoat es posterior a la de estas autoras, pero continúa con este recorrido comprendiendo al trabajo desde una concepción ampliada del mismo y asignándole un rol fundamental no solo con relación al modo de producción capitalista, sino también al modo de producción doméstico. Así, para Kergoat, el trabajo y su división entre varones y mujeres será el que determine los regímenes de opresión que las mujeres experimentan, tanto en el modo de producción doméstico como en el capitalista.

\section{DiVISIÓN SEXUAL DEL TRABAJO Y RELACIONES SOCIALES ESTRUCTURALES}

En la actualidad, la división sexual del trabajo es un concepto que se utiliza en una gran cantidad de estudios, sean demográficos, sociológicos o económicos. La economía feminista y la sociología con perspectiva de género han avanzado a pasos agigantados planteando la discusión de la importancia de considerar el trabajo doméstico dentro de los indicadores nacionales, el análisis censal y de la Encuesta Permanente de Hogares sobre la segregación laboral, los usos del tiempo, etc. (Wainerman 2012; Esquivel, 2012; Faur, 2014; Rodríguez Enríquez, 2015; entre otros).

A pesar de este amplio desarrollo, la utilización que muchas veces se hace de este concepto es a modo descriptivo, para designar las experiencias vivenciadas por las mujeres y las dificultades relacionadas con la doble jornada laboral, las tareas de cuidados, los puestos peor remunerados. ${ }^{4}$ En este sentido, la contextualización de dicho concepto en el interior de la corriente del FMF y su vinculación con el concepto de Relaciones Sociales Estructurales nos permite intentar acercarnos a otros usos y potencialidades del mismo, que no implican solamente definir un estado de cosas, sino situar a la división sexual del trabajo en el centro de la discusión acerca del poder que la clase de los varones ejerce sobre la clase de las mujeres.

Consideramos que avanzar en estas discusiones permitirá introducir nuevos interrogantes y reflexionar acerca de algunas problemáticas, como la llamada "crisis de los cuidados". Para ello retomamos los trabajos de Danièle Kergoat, socióloga del trabajo francesa, quien se ha dedicado a estudiar la división sexual del trabajo y las relaciones sociales estructurales de sexo en el mundo del trabajo y ha reflexionado acerca del lugar de las mujeres en la organización del trabajo productivo y reproductivo.

A través del estudio de diferentes casos (obreras, enfermeras, etc.), Kergoat sostiene que el fundamento de la opresión de las mujeres por parte de los varones y su división en clases se basa en la organización y la división del trabajo, siendo este la piedra angular de las relaciones de sexo (Kergoat, 1984, citado en Pfefferkorn, 2007, p. 61). Según Kergoat, la construcción o la producción social de sexos estriba, ante todo, en una base material, en la organización y la división concreta del trabajo tal como se encuentra dentro de la familia y dentro del sistema productivo, articulada, claro está, con otras relaciones sociales, en primer lugar, con las relaciones de clase.

Su propuesta metodológica parte de la premisa de la imposibilidad de separar la esfera del trabajo remunerado de la doméstica ya que, además de ser ilusoria, esta división responde a las categorías de la dominación masculina. Para ello la autora se detiene a lo largo de su obra en discutir la importancia de pensar al trabajo desde una definición ampliada del mismo (la transformación de la sociedad y la naturaleza, y en el mismo movimiento la transformación de sí mismo) planteándolo como el concepto clave para pensar la situación de dominación de las mujeres por parte de los varones.

Así, esta definición rompe con la operación de reducción que asociaba las palabras “trabajo" y "explotación” a la esfera salarial y que asociaba la emancipación solamente con la superación de la contradicción capital/ trabajo.

En palabras de Kergoat:

la división sexual del trabajo es la forma de división del trabajo social que se desprende de las relaciones sociales de sexo, histórica y socialmente modulada. Tiene como característica la asignación prioritaria de los hombres a la esfera productiva y de las mujeres a la esfera reproductiva así como, simultáneamente, la captación por parte de los hombres de las funciones con fuerte valor social agregado (políticas, religiosas, militares, etc.) (2002, p. 33). 
Esta división "tiene dos principios organizadores: el principio de separación (hay trabajos de hombres y trabajos de mujeres) y el principio jerárquico (un trabajo de hombre "vale" más que un trabajo de mujer)" (Kergoat, 2002, p. 64).

Por ende, la diferencia en la inserción de las mujeres en el mercado laboral no surge por un factor preferencial o biológico, sino que se puede explicar por una relación social de dominación donde las mujeres son asignadas a las tareas domésticas y de cuidado por fuera de la esfera de la producción asalariada, mientras que los varones son quienes captan las funciones con fuerte valor social agregado.

En esta división y en la asignación de roles entran en juego las cualidades llamadas "naturales" para los distintos sexos, entre las cuales algunas (las asignadas a los varones) son más valoradas que otras (las asignadas a las mujeres). Frente a esto, la autora se detiene en varias de sus investigaciones a analizar este proceso de legitimación de las cualificaciones, señalando que mientras que la calificación masculina (individual y colectiva) se construye socialmente, las cualidades que remiten al individuo o al género femenino se adquieren mediante un aprendizaje vivido erróneamente como individual, por el hecho de que se efectúa en la llamada esfera de lo privado y, por esa razón, son consideradas como innatas y naturales (Pfefferkorn, 2007).

Es así que en su reconocido texto "Las obreras" (1982), Kergoat menciona cómo la patronal justifica la contratación de mujeres debido a sus "dedos de hadas que manipulan mejor las piezas minúsculas de los componentes electrónicos" (Kergoat, 1982, p. 54) o cómo argumentan la elección de obreros especializados en ambos sexos:

Si hay varones especializados es porque la organización técnica lo exige, sin embargo, dentro de las fábricas de mujeres, su situación de obrera especializada se justifica por el hecho de que es un trabajo que conviene perfectamente a la naturaleza femenina (Kergoat, 1982, p. 56).

Basándose en las discusiones sobre la división sexual del trabajo, la autora enuncia como necesaria la incorporación del análisis de la globalidad del trabajo (productivo y reproductivo, asalariado y doméstico) no solo en el caso de las mujeres, sino también en el caso de los varones, para abrir el camino hacia un análisis sexuado de las prácticas sociales. Ello implica dejar de considerar al universo de la producción como el único universo referencial posible para los varones, error metodológico que aún hoy muchas veces se sigue repitiendo.

Desde la perspectiva de Kergoat, hablar en términos de división sexual del trabajo significa articular la descripción de los datos analizados en los casos empíricos (la contrastación de cómo se da esta división sexual del trabajo) con una reflexión sobre los procesos por los cuales la sociedad utiliza esta diferenciación para jerarquizar las actividades. Hablar en términos de división sexual del trabajo significa, entonces, reflexionar acerca de cómo se distribuye y ejerce el poder (Galerand y Kergoat, 2014).

\section{Relaciones Sociales estructurales de SeXo}

Antes de adentrarnos en su definición, es interesante detenernos en mencionar las problemáticas de traducción que ha presentado el concepto francés rapports sociaux de sexe.

La especificidad conceptual de la palabra rapport no tiene traducción exacta en el español, ya que en francés no denota solamente una relación, es decir un vínculo entre dos partes, sino que también explicita que dicha relación es asimétrica entre dos sujetos y, por tanto, comporta una dominación (Kergoat, 2002). Estas relaciones sexuales de dominación no se expresan solo en el nivel económico, sino que también abarcan el plano político y social.

Así, mientras que relations se utiliza para hablar de vínculos interindividuales, rapports remite a un plano estructural. Jules Falquet explica la diferencia entre relations y rapports distinguiendo un nivel microsocial y macroestructural, respectivamente (Falquet, 2017). Siguiendo a Falquet, Bolla (2020) presenta como solución ante este problema el mencionarlo como relaciones sociales estructurales (RSE), para poder 
incorporar el carácter de dominación al interior de las mismas. Xavier Dunezat (2017) en su texto en español utiliza la palabra rapports sociaux, y en las traducciones que se encuentran de los textos de Kergoat (Hirata y Kergoat, 1997) son mencionadas sólo como relaciones sociales de sexo. En este texto utilizaremos la propuesta de Falquet que retoma Bolla, al parecernos la que mejor logra reflejar la especificidad del concepto.

Hecha esta aclaración, pasamos a definir las RSE como una tensión que atraviesa el campo social y que erige ciertos fenómenos sociales en meollos (enjeux), en torno a los cuales se construyen grupos con intereses antagónicos (Kergoat, 2017, citado en Bolla, 2020). A decir de Dunezat, se suele considerar que tres RSE son más relevantes que otras, estas son las de sexo, raza y clase, lo que se explica por dos razones. En primer lugar, porque ningún campo social escapa a los procesos de categorización relacionados con estas RSE y, en segundo lugar, porque funcionan como RSE de producción (Godelier, 1984, citado en Dunezat, 2017). ${ }^{5}$

La distinción entre rapport y relación permite distinguir, así, dos niveles de análisis. Por un lado, las relaciones sociales son las relaciones concretas que establecen los grupos y los individuos. Estas relaciones están inscriptas dentro de las rapports sociaux (RSE) más generales, que son las que impactarán e influirán en las relaciones concretas que los individuos de diferentes clases establezcan entre sí, e incluso al interior de la misma clase. Esto nos permite hablar de sujetos, que actúan y son a la vez actuados por estas relaciones sociales, construyendo sus vidas a través de las prácticas sociales (Pfefferkorn, 2007).

A través de la conceptualización de las RSE de sexo se genera una vinculación entre las dos esferas (estructura familiar y sistema productivo), que fue necesaria para poder comprender la totalidad de la experiencia del trabajo, reconociendo que la explotación capitalista no termina cuando la mujer ingresa al hogar, y que la explotación de género no lo hace cuando la mujer entra a la fábrica.

Las características que poseen estas RSE son las de ser dinámicas (se modifican a lo largo del tiempo y el lugar) y ser consustanciales. Este concepto de consustancialidad o coextensión significa que no son disociables unas de otras, sino que existen anudadas de modo que solo puede distinguirse analíticamente ya que forman (de ahí el término) una única "sustancia”. Además, esto significa que están en un estado de interpenetración constante, lo que ocasiona que la opresión de la clase de las mujeres se haga más compleja a medida que se imbrica con otras formas de opresión.

Kergoat se separa del concepto de transversalidad y de las formas en la que este concepto ha sido utilizado teóricamente, ya que plantea que esta corriente piensa en términos de categorías que son estancas, mientras que desde la teoría de las RSE se propone pensar en términos de relaciones sociales de dominación, dinámicas y particulares de cada caso. A decir de la autora:

Las obreras no eran obreros, sus luchas como sus condiciones de trabajo, revelaban y producían una "otra" clase obrera. Pero simultáneamente, las obreras no eran mujeres. Quiero decir con esto que ni la categoría de obreros, ni la categoría de mujeres, mucho menos la adición de éstas, expresaban la realidad de la situación de trabajo concreto de las obreras, ni sus desplazamientos en el mercado de trabajo, ni sus prácticas de resistencia. Eso es lo que permite la consustancialidad: pensar en conjunto las diferentes formas de la división del trabajo y las divisiones en el seno de una misma clase (Kergoat, 2016, p. 17).

Por todo lo antes desarrollado, coincidimos con Bolla (2020) cuando menciona que el pasaje hacia el concepto de relaciones sociales estructurales de sexo muestra el recorrido teórico del feminismo materialista francés.

Consideramos que la potencialidad de este enfoque permite, en primer lugar, romper con el biologicismo, ya que los sexos no son más categorías fijas, inmutables, ahistóricas y asociales. Esto es así porque el trabajo y su división sexual genera estas clases sociales de sexo entre varones y mujeres. La clase de las mujeres deberá ser analizada como la clase de los individuos sobre quienes recae el trabajo doméstico y quienes quedan por fuera de las tareas con un fuerte valor social; mientras que la clase de los varones será la de quienes se encuentran mayoritariamente en el mercado laboral pago, quienes se aprovechan de las tareas realizadas por la clase de las mujeres y, por último, quienes capturan para sí las tareas con un fuerte valor social.

No obstante, este análisis quedaría trunco si no pensamos la imbricación de las diferentes relaciones sociales de dominación de manera situada e histórica. Es decir, qué significa ser una mujer, obrera, blanca en Francia 
de los años '80 (Kergoat, 1982) o qué implica ser una mujer, migrante, empleada doméstica en la Francia de los años 2010 (Kergoat, 2016). Este análisis situado e imbricacional permite romper con el universalismo y el ahistoricismo. Cada forma de organización tiene su expresión en términos de relaciones sociales de dominación que hay que comprender y analizar. Tomar en cuenta la diferenciación entre la actividad de las mujeres y de los varones permite comprender las modificaciones y variaciones históricas de las mismas que luego se plasman y son legitimadas por las instituciones, como el Estado, el derecho, el trabajo, los sindicatos, la familia, etc. (Pfefferkorn, 2017).

Por último, pensar la aplicación teórica en base a los clivajes relacionados con las RSE, nos permite poner la centralidad en el conflicto como parte dinámica de las relaciones sociales, lo que posibilita ir contra una concepción estática de la estructura social ya que relación (en tanto que rapport) significa, en efecto, contradicción, antagonismo, lucha por el poder, resistencia a considerar que los sistemas dominantes (capitalismo, patriarcado) son totalmente determinantes y que las prácticas sociales solo reflejan estas determinaciones (Hirata y Kergoat, 1997). Como ejemplo de este caso podemos mencionar el análisis que realiza Kergoat (2016) en relación con el trabajo de cuidado, donde plantea que se generan al menos tres relaciones de dominación. La primera es de clase, en la cual las mujeres del norte emplean a las mujeres que trabajan en el sector del cuidado (migrantes o no). La segunda es de competencia entre mujeres dentro del sector del trabajo de cuidados, todas precarias, aunque distintamente precarias, donde también entran en juego las relaciones étnicas.

En cuanto a la tercera relación, la de género, ésta se presenta a través de la externalización del trabajo doméstico. Su función es la de reducir las tensiones en las parejas heterosexuales profesionales (inter-sexo) en relación con la realización de las tareas domésticas y a la vez permitir una mayor flexibilidad de las mujeres en el mercado laboral con relación a la demanda de mayor tiempo de trabajo por parte de las empresas. En un nivel más macro, todo ello ocasiona que no se pueda dar una verdadera reflexión sobre las tareas de cuidados; ni se piense como alternativa una redistribución de las tareas entre quienes integran la pareja heterosexual.

En este sentido, a la hora de pensar las formas de superar dialécticamente la dicotomía entre la clase de las mujeres y la clase de los varones, la propuesta presenta una utilidad no solo teórica sino también práctica: las desigualdades de género en el interior del mundo del trabajo no son una "consecuencia no deseada" del proceso de organización laboral. Al contrario, esta jerarquización y segregación de los trabajos siguiendo un patrón de género es el eje fundamental de las mismas.

De ahí que lo que actualmente se menciona como "crisis de las tareas de cuidados" es la crisis de la división sexual del trabajo en el modo capitalista, donde el ingreso de mujeres al trabajo asalariado se realiza o bien tensionando las relaciones sociales estructurales de sexo, o bien incluyendo a otra mujer que realice el trabajo doméstico por aquella, lo que configura la explotación en el interior de la clase de las mujeres y las llamadas “cadenas globales de cuidado" (Kergoat, 2016).

\section{Pandemia, cuidados y trabajo asalariado. Reflexiones finales}

Realizar un análisis detallado de la aplicación de estas teorías a un caso concreto excedería la extensión y el objetivo de este trabajo. Por ello, nos limitaremos a presentar una serie de reflexiones en torno a la conciliación entre trabajo de cuidados y trabajo asalariado, que vincularemos con algunos resultados que se han presentado en relación con la pandemia. El objetivo es sugerir algunos caminos de indagación posibles que muestran lo desarrollado hasta aquí y permiten vislumbrar algunas problemáticas centrales.

La discusión entre la implementación o no de las políticas de "conciliación trabajo/familia" es un tema que atraviesa muchas de las discusiones con relación a la sociología del trabajo. ${ }^{6}$ En términos concretos, es cierto que las mismas ayudan a las mujeres a poder trabajar asalariadamente y encargarse de la esfera doméstica sin perjuicio de su rendimiento profesional. Pero si nos ponemos a analizar cómo se estructura el mundo del trabajo asalariado podemos ver que toda la organización está pensada en términos masculinos (Acker, 
2006). Es decir, se asume que los trabajadores tienen a disposición alguien que realice las tareas domésticas y de cuidado de sus familiares, perteneciente a la clase de sexo de las mujeres.

Por esto mismo, al introducir a las mujeres en esta estructura laboral, ellas se encuentran con dificultades, ya que a pesar de estar dentro del mercado laboral deben hacerse cargo de su "parte" en la división sexual del trabajo. Así es que las políticas de conciliación, que pretenden que las mujeres realicen ambos trabajos de manera armónica, lo que realmente ocasionan es una sobreexplotación de la clase de las mujeres o una explotación inter-sexo debido a la contratación de otras mujeres para encargarse de estas tareas. Quienes resultan beneficiados por estas políticas son, por un lado, los capitalistas y, por otro lado, la clase de los varones, ya que tales medidas no atacan las bases materiales de la opresión de la clase social de las mujeres, es decir, la división sexual del trabajo.

Resulta interesante, para terminar de ejemplificar esta idea, retomar algunos estudios realizados recientemente con relación a la productividad en el interior del mundo académico y las modificaciones que ha sufrido, debido a la situación de pandemia y de aislamiento obligatorio que se ha dado en diversos países. Los estudios de Cui et al. (2020) o de King y Frederickson (2020) acerca de la publicación de preprints por parte de varones y mujeres en contexto de la pandemia de COVID-19, demuestran que durante el confinamiento hubo una clara reducción de la presentación de los mismos por parte de mujeres frente a sus colegas varones. ${ }^{7}$ Esto se debe a que la pandemia enfrentó a este sector, que recaía fuertemente en instituciones formales o informales para las tareas de cuidados, con la imposibilidad de su utilización y los enfrentó, como mencionan Minello et al. (2020), a una "reorganización a corto término de los tiempos de cuidado y de trabajo", en la cual los grandes ganadores fueron los académicos varones.

En este caso, podemos ver que ante la imposibilidad de conseguir los servicios de cuidado por parte del mercado o del Estado, ni tampoco en redes familiares extendidas debido a las medidas de aislamiento tomadas por los países, estas familias debieron reestructurar su propio tiempo para poder hacerse cargo de las tareas domésticas y de cuidado (King y Frederikson, 2020). Es decir que, ante la no modificación de la división sexual del trabajo, fueron las mujeres quienes terminaron realizando la mayor cantidad de trabajo doméstico en comparación con sus colegas varones, lo que ha impactado (e impactará a futuro) fuertemente en su productividad académica.

Según la teoría de las RSE, sólo cuando se rompa verdaderamente con la división sexual del trabajo se podrá hablar de una sociedad donde no haya dominación de la clase de las mujeres por parte de la clase de los varones. Las propuestas de conciliación entre trabajo doméstico y trabajo asalariado no tienen en cuenta que toda la organización del trabajo asalariado se asienta sobre una estructura masculina que asume que el trabajo doméstico estará asegurado por la clase de las mujeres. Los casos anteriormente mencionados nos permiten dar cuenta de la importancia de reflexionar acerca de la división sexual del trabajo como eje de opresión de la clase social de las mujeres y de generar líneas de acción para avanzar hacia una sociedad donde estas tareas se repartan más equitativamente.

\section{REFERENCIAS}

Acker, J. (2006). Inequality Regimes - Gender, Class, and Race in Organizations. Gender \& Society, 20, 441-64.

Bolla, L. (2016). Sobre la teoría y la práctica feminista: una aproximación al feminismo materialista. Trabajo presentado en $I X$ Jornadas de Sociología de la UNLP, Universidad Nacional de La Plata, Ensenada, Argentina. Recuperado de http://www.memoria.fahce.unlp.edu.ar/trab_eventos/ev.9144/ev.9144.pdf

Bolla, L. (2018). Cartografías feministas materialistas: relecturas heterodoxas del marxismo. Nómadas, 48, 117-134. Recuperado de http://www.memoria.fahce.unlp.edu.ar/art_revistas/pr.8977/pr.8977.pdf

Bolla, L. (2020). La naturaleza del sexo: Relecturas sintomáticas del feminismo materialista (Tesis doctoral inédita). Universidad Nacional de La Plata, Facultad de Humanidades y Ciencias de la Educación, Ensenada, Argentina. 
Cui, R. Ding, H. y Zhu, F. (2020). Gender Inequality in Research Productivity During the COVID-19 Pandemic. Recuperado de https://ssrn.com/abstract $=3623492$

Curiel, O. y Falquet, J. (Comps.). (2005). Elpatriarcado al desnudo: Tres feministas materialistas. Buenos Aires: Brecha Lésbica. Recuperado de https://julesfalquet.files.wordpress.com/2010/06/el-patriarcado-al-desnudo-tres-femi nistas-materialistas2.pdf

Delphy, C. (2013). L'ennemi principal. Vol. 1 Économie politique du patriarcat y Vol. II Penser le genre. París: Syllepse.

Dunezat, X. (2017). Sexo, raza, clase y etnografía de los movimientos sociales. Herramientas metodológicas para una perspectiva interseccional. Revista de Investigaciones Feministas, 8(1), 95-114.

Esquivel, V. (2012). Introducción: Hacia una economía feminista desde América Latina. En Esquivel et. al. La Economia feminista desde América Latina: una hoja de ruta sobre los debates actuales en la región (pp. 24-41). Santo Domingo: ONU-Mujeres.

Estermann, V. (2018). ¿Modo de producción capitalista o doméstica? Cómo pensar el trabajo desde la perspectiva del feminismo materialista francófono. Trabajo presentado en X Jornadas de Sociología de la UNLP, Universidad Nacional de La Plata, Ensenada, Argentina. Recuperado de http://jornadassociologia.fahce.unlp.edu.ar/x-jorna das/actas/EstermannPONmesa35revisada.pdf

Falquet, J., Petteni, O. y Wustefeld, S. (2016). Entretien avec Jules Falquet: Matérialisme féministe, crise du travail salarié et imbrication des rapports sociaux. Cahiers du GRM (Groupe de Recherches Matérialistes), 10. https:// doi.org/10.4000/grm.839

Falquet, J. (2017). La combinatoria straight. Raza, clase, sexo y economía política: análisis feministas materialistas y decoloniales. Descentrada, 1(1), e005. Recuperado de http://www.descentrada.fahce.unlp.edu.ar/article/view/ DESe005

Faur, E. (2014). El cuidado infantil en el siglo XXI. Mujeres malabaristas en una sociedad desigual. Buenos Aires: Siglo XXI Editorial

Femenías, M. L. (2015). El feminismo materialista francés en el marco general de las teorías feministas y de género. Mora, 21, 149-163. https://doi.org/10.34096/mora.n21.2406

Galerand, E. y Kergoat, D. (2014). Les apports de la sociologie du genre à la critique du travail. La nouvelle revue du travail, 4. Recuperado de http://journals.openedition.org/nrt/1533

Guillaumin, C. (2005). Práctica del poder e idea de Naturaleza. En O. Curiel y J. Falquet. (Comps.). Elpatriarcado al desnudo: tres feministas materialistas (pp. 19-56), Buenos Aires: Brecha Lésbica.

Hirata, H. y Kergoat, D. (1997). La división sexual del trabajo: permanencia y cambio. Buenos Aires: Asociación, Trabajo y Sociedad.

Kandel, E. (2006). División sexual del trabajo ayer y hoy: una aproximación al tema. Buenos Aires: Dunken

Kergoat, D. (1982). Les ouvrières. Paris: Le Sycomore.

Kergoat, D. (2002). Le rapport social de sexe. De la reproduction des rapports sociaux à leur subversion. Actuel Marx, $2(30), 85-100$.

Kergoat, D. (2016). Le care et l'imbrication des rapports sociaux. En N. Araujo Guimarães, M. Maruani y B. Sorj. (Eds.). Genre, race, clase. Travailler en France et au Brésil. Paris: L'Harmattan.

Kergoat, D. (2017). Division sexuelle du travail et rapports sociaux de sexe. En H. Hirata, F. Laborie, H. Le Doaré y D. Senotier. (Coords.). Dictionnaire critique du féminisme (pp. 35-44). Paris: PUF.K.

King, M. M. y Frederickson, M. (2020). The Pandemic Penalty: The gendered effects of COVID-19 on scientific productivity. https://doi.org/10.31235/osf.io/8hp7m

Minello, A., Martucci, S. y Manzo, L. (2020). The pandemic and the academic mothers: present hardships and future perspectives. European Societies: European Societies in the Time of the Coronavirus Crisis, 23(1). https://doi.org /10.1080/14616696.2020.1809690

Pasero Brozovich, M. (2016). Reflexiones feministas sobre el trabajo: recorridos desde el Feminismo Materialista. Trabajo presentado en $V$ Encuentro Latinoamericano de Metodología de las Ciencias Sociales, Mendoza, Argentina. Recuperado de http://www.memoria.fahce.unlp.edu.ar/trab_eventos/ev.8543/ev.8543.pdf 
Pfefferkorn, R. (2007). El trabajo, un eje fundamental de las relaciones sociales de sexo. Revista de Ciencias Sociales (Cl), 18, 53-70.

Rodríguez Enríquez, C. (2011). Programas de transferencias condicionadas de ingreso e igualdad de género ¿Por dónde anda América Latina?. Serie Mujer y Desarrollo, 109, Chile: CEPAL. División Asuntos de Género.

Rodríguez Enríquez, C. (2015). Economía feminista y economía del cuidado. Nueva Sociedad, 256, 30-44.

Wainerman, C. (2012). Los usos del tiempo de trabajo y la división del trabajo según género. En A. Domínguez Mon, A. M. Méndez Diz; P. Schwartz y M. Camejo (comps.). Usos del tiempo, temporalidades y géneros en contextos (pp. 49-54). Buenos Aires: Antropofagia.

\section{Notas}

1 Cuando utilicemos las palabras clase, raza y sexo, haremos referencia a las relaciones sociales de dominación que crean estas categorías, y no a algo que existe natural o biológicamente. Debido a que son las categorías que se elaboran desde el Feminismo Materialista Francés (FMF) decidimos mantenerlas como tal, en vez de reemplazarlas por otras como género o etnia.

2 Se trata de una clase social que no se encuentra cultural ni biológicamente determinada, como se verá más adelante.

3 Para un mayor desarrollo de las propuestas del FMF, ver Bolla (2018; 2020); Pasero Brozovich (2016); Estermann (2018) entre otros.

4 Una gran excepción, entre otras, es el trabajo de Kandel (2006).

5 También hay debates sobre la relación ontológica entre los tres rapports sociaux y otros ejes de opresión (cf. Dunezat, 2017).

6 En el caso de las políticas de "conciliación trabajo/familia" en los lugares de trabajo, las mismas proponen no solo flexibilizar tiempos de llegada y de salida, posibilitar una mayor cantidad de días por maternidad o por enfermedad de familiares, sino también pensar al teletrabajo y la flexibilización como alternativas a ser tomadas en cuenta, para abordar estas problemáticas.

7 Los preprints son versiones manuscritas de publicaciones antes de su revisión por pares (antes de su certificación como publicaciones formales en una revista académica). Su publicación permite no solo aumentar la fluidez del intercambio de resultados científicos, ya que los procesos de publicación suelen llevar tiempo, sino evitar problemas de robo de datos y plagios. 